\title{
Quelle est la part des taxes dans le prix d'une boisson alcoolisée type au Canada et pourquoi est-ce important?
}

\section{Samuel Churchill, M. Sc.; Tim Stockwell, Ph. D.; Adam Sherk, Ph. D.}

Cet article a fait l'objet d'une évaluation par les pairs.

\section{Résumé}

Introduction. Les distillateurs et les brasseurs canadiens ont affirmé que les taxes gouvernementales représentent entre $50 \%$ et $80 \%$ du prix des boissons alcoolisées. Ces affirmations ont été formulées dans le cadre de campagnes visant à réduire les taxes sur l'alcool.

Méthodologie. Nous avons examiné le bien-fondé de ces affirmations au moyen des données de Santé Canada accessibles au public ainsi que des données provinciales sur les ventes par produit et la ventilation des prix des boissons alcoolisées types dans les principaux secteurs de marché.

Résultats. Dans tous les cas, les taux de taxe de vente totale et de taxe d'accise se situent généralement entre $20 \%$ et $30 \%$ du prix de détail final, soit bien en deçà de ce qu'avancent les représentants de l'industrie.

Mots-clés : consommation de substances, consommation d'alcool, réduction des méfaits, enquêtes, santé publique, coûts, coûts sociétaux, coûts et méfaits de l'usage de substances au Canada, fiscalité

\section{Introduction}

Les évaluations scientifiques approfondies des politiques gouvernementales visant à réduire la consommation d'alcool et les méfaits qui y sont liés concluent systématiquement que les stratégies fiscales et de fixation des prix sont les plus efficaces ${ }^{1-3}$. Selon une évaluation officielle récente, la plupart des gouvernements canadiens n'ont pas réussi à mettre en œuvre des politiques fondées sur des données probantes en ce qui concerne l'alcool et ont de piètres stratégies fiscales et de fixation des prix ${ }^{4}$.

En 2017, le gouvernement fédéral a réintroduit l'indexation de la taxe d'accise sur l'alcool (afin que la valeur de la taxe d'accise suive l'évolution de l'inflation). Cependant, il ressort d'une évaluation de la mise en œuvre de cette politique que, dans l'ensemble, le niveau et la structure de la taxe d'accise sont relativement peu efficaces en matière d'amélioration des résultats pour la santé et la sécurité publiques $^{5}$. Les lacunes majeures en sont de faibles taux de taxe, qui témoignent de l'absence d'indexation des taxes en fonction du coût de la vie, une incapacité à taxer la plupart des boissons alcoolisées en fonction de leur teneur en alcool et enfin une application inégale des taux de taxe en fonction des types de boissons.

Les données probantes scientifiques en faveur de l'utilisation de stratégies fiscales et de fixation des prix pour réduire les méfaits liés à la consommation d'alcool sont solides. Néanmoins, ces stratégies doivent être présentées avec prudence, dans la mesure où elles sont souvent peu populaires $^{1}$.

Pour éviter la réintroduction de l'indexation des taxes d'accise, les producteurs canadiens ont soutenu que cette mesure impliquait une augmentation des taxes ${ }^{6}$.

\section{Points saillants}

- Les groupes de l'industrie des boissons alcoolisées ont exagéré le niveau de taxation appliqué sur les boissons alcoolisées types au Canada.

- Les distillateurs affirment que le taux de taxe est de $80 \%$, mais nos estimations montrent qu'il se situe entre $20 \%$ et $30 \%$.

- Les brasseurs affirment que le taux de taxe est de $47 \%$, mais nos estimations montrent qu'il se situe entre $16 \%$ et $18 \%$.

En réalité, l'indexation permettrait seulement de s'assurer que les prix suivent le rythme de l'inflation. Les distillateurs et les brasseurs canadiens ont affirmé que les taxes constituaient déjà $80 \%$ du prix des spiritueux 6 et $50 \%$ du prix de la bière?

Ces affirmations des producteurs sont publiques depuis 2018, et un examen indépendant des chiffres avancés est nécessaire. Les producteurs d'alcool s'opposent vraisemblablement aux augmentations des taxes d'accise parce que celles-ci entrent en concurrence directe avec leurs marges bénéficiaires et ont une influence sur le prix auquel ils peuvent vendre leurs produits aux détaillants et aux distributeurs. De plus, dans ses affirmations, l'industrie néglige de définir de façon rigoureuse ce qu'elle considère être des " taxes " pour les besoins de ses chiffres, laissant cette définition sujette à l'interprétation.

Les chiffres de l'industrie semblent incorporer les bénéfices liés au monopole des gouvernements sur les ventes au détail dans la définition de la taxation. Ce 
raisonnement implique des normes différentes pour les détaillants privés, qui effectuent la majeure partie des ventes d'alcool au Canada ${ }^{5}$, et les détaillants publics, et ne considère pas les bénéfices des détaillants privés comme des taxes.

Au moyen des données sur les ventes par produit des magasins de vins et spiritueux des gouvernements de la ColombieBritannique et de l'Ontario, nous avons enquêté sur la véracité des affirmations de l'industrie. Nous espérons que les résultats de nos analyses feront la lumière sur ce qui est payé par les clients. Ces analyses pourraient également aider les décideurs à relativiser les affirmations de l'industrie des boissons alcoolisées lors d'une révision potentielle des réformes des prix et des taxes sur les boissons alcoolisées.

\section{Méthodologie}

Pour examiner la ventilation des prix et des taxes, nous avons consulté les statistiques agrégées fournies par Statistique Canada $^{8,9,10}$ ainsi que les données sur les ventes par produit des magasins de vins et spiritueux de la Colombie-Britannique et de l'Ontario, qui nous ont été envoyées directement par les autorités provinciales régissant les boissons alcoolisées. Les données de la Colombie-Britannique remontent à 2014 et 2016, et les données de l'Ontario, à 2017. Voici la synthèse des paramètres que nous avons établis à partir des ensembles de données sur les ventes de produits :

- Le format type d'une boisson dans chaque grande catégorie de boissons (la bière, le vin, les spiritueux, les panachés et les cidres), qui a été choisi car il correspond au format le plus souvent vendu en matière de ventes totales en dollars et de ventes totales en litres de boisson.

- Les valeurs moyennes (pondérées en fonction des ventes totales en dollars) du prix, de la teneur en alcool, de la taxe de vente et de la taxe d'accise ad valorem payées pour chaque format de boisson type en Colombie-Britannique et en Ontario, valeurs à l'échelle de la province qui ont ensuite été combinées au moyen d'une autre moyenne pondérée, cette fois en fonction de la population, afin de fournir une estimation nationale.
Nous avons pris en compte quatre grands marchés de boissons (bière, vin, spiritueux et cidres/panachés) lors de l'agrégation des données de Statistiques Canada, mais nous avons séparé les cidres et les panachés en deux catégories pour l'examen des données par produit pour la ColombieBritannique et l'Ontario.

Pour estimer, au sein de chaque marché, les proportions correspondant aux coûts de gros du fabricant, aux taxes du gouvernement et aux bénéfices de l'autorité responsable de l'alcool à l'aide des données de Statistique Canada, nous avons combiné une estimation directe des proportions fondées sur les données de Statistique Canada à des méthodes utilisées antérieurement pour estimer le fardeau de la taxe d'accise sur les boissons alcoolisées au Canada ${ }^{11}$. Les coûts de gros, les taxes de vente ad valorem et les bénéfices ${ }^{9}$ ont été distribués en fonction de la part de marché occupée par chaque catégorie de boisson ${ }^{8,9}$. Les taxes d'accise ont été distribuées en utilisant des modèles décrits précédemment ${ }^{11}$, en considérant que la taxe d'accise et les changements à la taxe d'accise étaient entièrement transférés aux consommateurs ${ }^{12}$. Ces modèles s’appuient sur les données détaillées en ce qui concerne les prix des produits individuels, les ventes et la production et ils tiennent également compte des différents taux de taxe pour des brasseurs ayant des volumes de production faibles, moyens et élevés. Afin de présenter des résultats qui sont généralisables à l'échelle du Canada, nous avons tenu compte uniquement des taux de taxe représentatifs qui sont disponibles à un niveau agrégé, soit la taxe d'accise, la taxe fédérale sur les produits et services (TPS) et les taux de taxe de vente provinciale (TVP) de la ColombieBritannique et de l'Ontario. Une approche qui identifie un marché précis, par exemple l'imposition additionnelle ad valorem de $25 \%$ sur les ventes d'alcool à l'île-duPrince-Édouard et la taxe de base sur l'alcool de l'Ontario sur les boissons produites en Ontario, pourrait produire des résultats différents mais serait nettement moins généralisable à l'ensemble du Canada.

\section{Résultats}

Au sein de chaque grand marché de boisson, le taux global de taxe varie entre $20 \%$ et $31 \%$, le taux le plus élevé étant appliqué aux spiritueux (tableau 1). Le prix de gros forme quant à lui entre environ $37 \%$ à $42 \%$ du prix de détail final et les bénéfices totaux de l'autorité responsable de l'alcool forment le reste, soit entre $32 \%$ et $38 \%$.

Dans le cas d'une bouteille de spiritueux, boisson qui présente la charge fiscale la plus élevée en proportion du prix total, la part de taxe totale moyenne est de $8,92 \$$ sur un prix de 43,01 \$, soit environ 20,7\% (tableau 2). Des calculs similaires effectués pour le reste des boissons conduisent à l'estimation d'une charge fiscale de moins de $20 \%$ pour l'ensemble des autres types de boissons. Il convient de souligner que les taxes de vente ad valorem forment la grande majorité des taxes visées par l'examen, la taxe d'accise constituant une très faible part du prix total des boissons alcoolisées autres que les spiritueux. Le taux le plus élevé de taxe d'accise, en pourcentage du prix final, concerne les spiritueux, et les taux de ces taxes d'excise varient en fonction de la teneur en alcool et du volume.

\section{Analyse}

Nos résultats offrent une synthèse simple des taxes habituelles incluses dans le prix

TABLEAU 1

Part des taxes, en pourcentage, dans le prix de détail final des boissons alcoolisées «types» au Canada, 2017-2018, données agrégées

\begin{tabular}{lccccc} 
& \multicolumn{5}{c}{ Part en pourcentage (\%) } \\
\cline { 2 - 6 } Boisson & Prix de gros & Taxe d'accise & Bénéfices & $\begin{array}{c}\text { Taxe de } \\
\text { vente }\end{array}$ & $\begin{array}{c}\text { Taxe d'accise + taxe } \\
\text { de vente }\end{array}$ \\
\hline Bière & 41,35 & 8,90 & 36,93 & 12,81 & 21,71 \\
Vin & 42,26 & 6,91 & 37,74 & 13,09 & 20,00 \\
Spiritueux & 36,67 & 19,22 & 32,75 & 11,36 & 30,58 \\
$\begin{array}{l}\text { Panachés/ } \\
\text { cidres }\end{array}$ & 41,21 & 9,22 & 36,80 & 12,77 & 21,99 \\
\hline
\end{tabular}

Source des données : Statistique Canada. Tableaux 10-10-0011-01, 10-10-0010-01 et 10-10-0012-01. Ottawa (Ont.) : Statistique Canada; $2018^{8-10}$. 
TABLEAU 2

Prix moyens et taxes payées pour un format type de boisson en Colombie-Britannique et en Ontario, en fonction du type de boisson

\begin{tabular}{lccccccc} 
Boisson & $\begin{array}{c}\text { Volume } \\
(\mathbf{m l})\end{array}$ & $\begin{array}{c}\text { Teneur } \\
\text { moyenne } \\
\text { en alcool } \\
(\%)\end{array}$ & $\begin{array}{c}\text { Prix } \\
\text { moyen }(\$)\end{array}$ & $\begin{array}{c}\text { Taxe de } \\
\text { vente } \\
\text { moyenne } \\
(\$)\end{array}$ & $\begin{array}{c}\text { Taxe } \\
\text { d'accise } \\
\text { moyenne } \\
(\$)\end{array}$ & $\begin{array}{c}\text { Taxe } \\
\text { Montant } \\
\text { restant }(\$)\end{array}$ & $\begin{array}{c}\text { Toyenne } \\
\text { totale } \\
\text { moys }\end{array}$ \\
\hline Bière $^{\mathrm{a}}$ & 473 & $5,07^{\mathrm{c}}$ & 2,41 & 0,31 & 0,06 & 2,03 & 0,38 \\
Bière $^{\mathrm{b}}$ & 2130 & $4,93^{\mathrm{c}}$ & 10,93 & 1,42 & 0,54 & 8,97 & 1,96 \\
Vin & 750 & 15,05 & 19,88 & 2,59 & 0,46 & 16,83 & 3,05 \\
Spiritueux & 750 & 37,86 & 43,01 & 5,60 & 3,32 & 34,09 & 8,92 \\
Panachés & 473 & 6,46 & 2,91 & 0,38 & 0,14 & 2,39 & 0,52 \\
Cidres & 500 & 4,71 & 2,94 & 0,38 & 0,15 & 2,41 & 0,53 \\
\hline
\end{tabular}

Remarque : Les données dans le tableau ont été agrégées à partir des données provinciales sur les ventes par produit.

${ }^{a}$ Une canette de bière

${ }^{\mathrm{b}}$ Une caisse de six bouteilles de $355 \mathrm{ml}$ chacune

' La moyenne de teneur en alcool est calculée en fonction de boissons dans la même catégorie de volume. La catégorie de $473 \mathrm{~mL}$ contenait davantage de bières plus fortes produites en masse que la catégorie de $2130 \mathrm{~mL}$, laquelle contenait davantage de bières artisanales, ce qui explique la différence dans la teneur moyenne en alcool.

des boissons alcoolisées au Canada. Ils sont interprétables à l'échelle nationale. Lors de l'application de cette analyse à une province en particulier, les taux de taxe sur l'alcool de cette province (comme la taxe de base sur l'alcool de l'Ontario sur les boissons produites en Ontario ou la taxe de vente ad valorem additionnelle de $25 \%$ pour l'île-du-Prince-Édouard) doivent être pris en compte. Même si ces variations régionales avaient pour effet de doubler la charge fiscale dans une province donnée, tous les résultats seraient inférieurs aux charges fiscales que l'industrie prétend se voir imposer.

Les distillateurs canadiens affirment que les taxes représentent $80 \%$ du prix des spiritueux au Canada ${ }^{9}$. Or, selon nos estimations, la charge fiscale est plutôt de $20 \%$ à $30 \%$. De plus, la ventilation des prix au moyen des données agrégées de Statistique Canada indique que les distillateurs reçoivent plus du tiers du prix final des spiritueux qu'ils produisent. Ces données laissent entendre que les distillateurs canadiens doivent définir et étayer leurs affirmations avec plus de rigueur. Même si l'industrie inclut les bénéfices réalisés par les détaillants publics dans son calcul de $80 \%$ de taxe, les montants ne concordent pas avec ce qu'elle affirme.

Nos constatations viennent également contredire l'allégation des brasseurs selon laquelle près de la moitié du prix de la bière est constitué de taxes. Selon nos calculs, ces chiffres varient entre $16 \%$ et $18 \%$ des données par produit (tableau 2) et la charge fiscale estimée (taxe d'accise et taxe de vente) se chiffre à $22 \%$ (tableau 1 ). Ces chiffres sont fortement tributaires des taux de taxe provinciaux sur la vente des produits et de la taxe d'accise à taux variable qui est établie en fonction du volume de production de la brasserie.

Nous avons constaté que $41 \%$ du prix de vente des bières correspond au prix de gros (tableau 1). Est-il possible que l'industrie ait inclus les bénéfices réalisés par les détaillants publics dans sa valeur calculée de $50 \%$ de taxe? Si c'est le cas, nous insistons sur le fait qu'une telle interprétation est erronée, car elle établit des normes différentes pour les détaillants privés et pour les détaillants publics.

Peu de recherches ont été menées au Canada sur la relation entre les taxes d'accise, les niveaux de consommation d'alcool et les méfaits liés à l'alcool. L'existence de ces relations et leur portée générale étant généralement bien établies dans la littérature internationale ${ }^{1-3,11}$, il serait utile de les quantifier de façon plus précise pour les marchés canadiens. Les études de modélisation sur les répercussions probables de l'utilisation de stratégies fiscales et de fixation des prix différentes sur les niveaux de consommation d'alcool et les méfaits liés à l'alcool au Canada ${ }^{11}$ peuvent se révéler utiles pour améliorer l'efficacité des politiques de santé publique visant à réduire les méfaits liés à l'alcool.

\section{Conclusion}

En résumé, le niveau de base actuel de taxation provinciale et fédérale sur les boissons alcoolisées au Canada est inférieur au tiers du prix de détail final pour tous les types de boissons alcoolisées, et inférieur à $22 \%$ pour la bière, le vin et les panachés/cidres. Ces analyses permettent de penser qu'il existe une grande latitude pour augmenter les taxes d'accise sur les produits alcoolisés au Canada. Cette mesure permettrait d'améliorer les résultats en matière de santé publique et de compenser les pertes de revenus découlant de l'absence d'indexation antérieure des taxes d'accise sur l'alcool en fonction du coût de la vie.

\section{Conflits d'intérêts}

Aucun.

\section{Contribution des auteurs et avis}

SC : Logiciel, méthodologie, rédaction de la première version du manuscrit; TM : Conceptualisation, méthodologie, rédaction (relecture et révision); AS : Méthodologie et rédaction (relecture et révision).

Le contenu de l'article et les points de vue qui y sont exprimés n'engagent que les auteurs; ils ne correspondent pas nécessairement à ceux du gouvernement du Canada.

\section{Références}

1. Babor TF, Caetano R, Casswell S, et al. Alcohol: No ordinary commodity research and public policy - Revised edition. Oxford (Royaume-Uni): Oxford University Press; 2010. https://doi .org/10.1093/acprof:oso/9780199551149 .001 .0001

2. Nelson TF, Xuan ZM, Babor TF, et al. Efficacy and the strength of evidence of U.S. alcohol control policies. Am J Prev Med. 2013;45(1):19-28. https:// doi.org/10.1016/j.amepre.2013.03.008

3. Organisation mondiale de la Santé. Combattre les MNT : " meilleurs choix » et autres interventions recommandées pour lutter contre les maladies non transmissibles. Genève $(\mathrm{CH})$ : Organisation mondiale de la Santé; 2017. Rapport $n^{\circ}$ WHO/NMH/NVI/17.9 
4. Stockwell T, Wettlaufer A, Vallance K, et al. Stratégies pour réduire les méfaits et les coûts liés à l'alcool au Canada : Une revue des politiques provinciales et territoriales [Internet]. Victoria (C.-B.) : Institut canadien de recherche sur l'usage de substances, Université de Victoria; 2019 [consultation le 12 juillet 2020]. En ligne à : https:// www.uvic.ca/research/centres/cisur /assets/docs/report-cape-pt-fr.pdf

5. Wettlaufer A, Vallance K, Chow C, et al. Stratégies pour réduire les méfaits et les coûts liés à l'alcool au Canada : Une revue des politiques fédérales [Internet]. Victoria (C.-B.) : Institut canadien de recherche sur l'usage de substances, Université de Victoria; 2019 [consultation le 12 juillet 2020]. En ligne à : https://www.uvic.ca/research /centres/cisur/assets/docs/report-cape -federales-fr.pdf

6. Spirits Canada. Not on my tab! $80 \%$ Tax on spirits and the government wants even more? [Internet]. Toronto (Ont.): Spirits Canada; [consultation le 12 juillet 2020]. En ligne à : https:// notonmytab.ca

7. Beer Canada. Report: Canada's beer taxes 5 times higher (and rising) than U.S. beer taxes [Internet]. Calgary (AB): Cision; 2018 May 07 [consultation le 12 juillet 2020]. En ligne à : https:// www.newswire.ca/news-releases /report-canadas-beer-taxes-5-times -higher-and-rising-than-us-beer -taxes-681916701.html

8. Statistique Canada. Tableau : 10-100011-01 (anciennement CANSIM 1830024) : Valeur des ventes de boissons alcoolisées des régies des alcools et d'autres points de vente au détail, selon le type de boisson ( $\times 1000)$ [Internet]. Ottawa (Ont.) : Statistique Canada; 2018 [consultation le 12 juillet 2020]. En ligne à : https://www150 .statcan.gc.ca/t1/tbl1/fr/tv.action?pid $=1010001101$

9. Statistique Canada. Tableau : 10-100010-01 (anciennement CANSIM 1830023) : Ventes de boissons alcoolisées des régies des alcools et d'autres points de vente au détail, selon la valeur, le volume et le volume absolu [Internet]. Ottawa (Ont.) : Statistique Canada; 2018 [consultation le 12 juillet 2020]. En ligne à : https://www150 .statcan.gc.ca/t1/tbl1/fr/tv.action?pid $=1010001001$
10. Statistique Canada. Tableau : 10-100012-01 (anciennement CANSIM 1830025) : Bénéfice net des régies des alcools et recettes des administrations publiques de la vente des boissons alcoolisées ( $\times 1000$ ) [Internet]. Ottawa (Ont.) : Statistique Canada; 2018 [consultation le 12 juillet 2020]. En ligne à : https://www150.statcan.gc.ca/t1 /tbl1/fr/tv.action?pid = 1010001201

11. Stockwell T, Churchill S, Sherk A, Sorge J, Gruenewald P. Réduction des décès et des hospitalisations dus à l'alcool grâce à des politiques fiscales et d'établissement des prix différentes? Modélisation des effets sur la consommation d'alcool, les revenus et les méfaits liés à l'alcool au Canada. Promotion de la santé et prévention des maladies chroniques au Canada. 2020;40(5-6):171-184. https://doi.org /10.24095/hpcdp.40.5/6.04f

12. Nelson JP, Moran JR. Effects of alcohol taxation on prices: a systematic review and meta-analysis of passthrough rates. BE J Econ Anal Policy. 2019;20(1):20190134. https://doi.org /10.1515/bejeap-2019-0134 